\title{
The Effect of Acromioplasty on the Critical Shoulder Angle and Acromial Index
}

\author{
Ian S. MacLean, M.D., Anirudh K. Gowd, M.D., Brian R. Waterman, M.D., \\ Ian J. Dempsey, M.D., M.B.A., Bernard R. Bach, M.D., Brian J. Cole, M.D., M.B.A., \\ Anthony A. Romeo, M.D., and Nikhil N. Verma, M.D.
}

\begin{abstract}
Purpose: To evaluate the effect of acromioplasty using a cutting block technique on bony coverage as measured by the critical shoulder angle (CSA) and acromial index (AI). Methods: This study is a retrospective radiographic review using data from a previous prospective randomized clinical trial that offered enrollment to patients aged 18 years or older with a full-thickness tear of the superior rotator cuff between October 2007 and January 2011. Each patient was allocated to repair with either acromioplasty using a cutting block technique or non-acromioplasty treatment arms in a blinded fashion. Medical and demographic information was recorded for each patient. Between January 2017 and December 2017, patients were contacted for repeat follow-up clinical evaluation and radiographs. Measurements of acromial index and critical shoulder angle were performed on pre- and postoperative radiographs by a single reviewer. Results: Seventyone $(75 \%)$ patients were available for follow up. The 2 groups were similar in terms of baseline demographics and acromial type. When compared with preoperative measures, acromioplasty did not result in significant reductions in mean CSA $\left(34.5^{\circ} \mathrm{vs}\right.$ $\left.35.5^{\circ} ; P=.293\right)$ or $\mathrm{AI}(0.68$ vs $0.66 ; P=.283)$. Furthermore, postoperative CSA $\left(34.5^{\circ}\right.$ vs $\left.36.2^{\circ}, P=.052\right)$ and $\mathrm{AI}(0.66$ vs 0.67 , $P=.535$ ) demonstrated no statistically significant differences between patients with and without acromioplasty, respectively. Conclusions: There was no statistically significant change in either the CSA or AI following acromioplasty, nor was there a significant postoperative difference in CSA or AI between the group that underwent acromioplasty and the group that did not. Clinical Relevance: Some studies suggest a greater postoperative CSA may result in greater risk of retear after arthroscopic rotator cuff repair. The CSA and AI may not be modifiable with acromioplasty.
\end{abstract}

A cromial morphology has been associated with the development and progression of rotator cuff tears (RCTs). ${ }^{1,2}$ Proposed descriptive systems range from the

From the Section of Sports Medicine (I.J.D., B.R.B., B.J.C., N.N.V.), Department of Orthopaedic Surgery (I.S.M., I.J.D., B.R.B., B.J.C., N.N.V.), Rush University Medical Center, Chicago, Illinois; Section of Sports Medicine (B.R.W.), Department of Orthopaedic Surgery (A.K.G., B.R.W.), Wake Forest Baptist Medical Center, Winston-Salem, North Carolina; and Section of Shoulder and Elbow Surgery, Rothman Institute, Philadelphia, Pennsylvania (A.A.R.), U.S.A.

The authors report the following potential conflicts of interest or sources of funding: B.J.C. reports other from Aesculap/B. Braun, American Journal of Orthopedics, American Journal of Sports Medicine, Aqua Boom, Arthrex, Arthroscopy, Arthroscopy Association of North America, Athletico, Biomerix, Cartilage, DJ Orthopaedics, Elsevier Publishing, Flexion, Geistlich, GiteliScope, International Cartilage Repair Society, Journal of Bone and Joint Surgery: American, Journal or Shoulder and Elbow Surgery, JRF Ortho, National Institutes of Health, Operative Techniques in Sports Medicine, Ossio, Regentis, Sanofi-Aventis, Smith $\theta$ Nephew, Tornier, and Zimmer, outside the submitted work. A.A.R. reports personal fees and other from Arthrex, non-financial support and other from Paragen Technology, other from SLACK Inc, other from Wolters Kluwer Health, and other from Saunders/Mosby-Elsevier, outside the submitted work. N.N.V. reports grants from Arthrex, other from Arthroscopy, grants from Breg, other from Cymedica, other from Minivasive, other from Omeros, other from Orthospace, categorical, such as the Bigliani acromial types, to the quantitative, like the critical shoulder angle (CSA) and acromion index $(\mathrm{AI})$. While the well-known Bigliani

grants from Ossur, grants and other from Smith $\theta$ Nephew, other from Vindico Medical-Orthopaedics Hyperguide, and grants from Wright Medical Technology, outside the submitted work. N.N.V. is a member of the editorial or governing boards of the American Orthopaedic Society for Sports Medicine, American Shoulder and Elbow Surgeons, Arthroscopy Association of North America, Knee, and SLACK Incorporated. B.R.W. reports AAOS: board or committee member; American Orthopaedic Society for Sports Medicine: board or committee member; Arthrex: research support; Arthroscopy: editorial or governing board; publishing royalties, financial or material support; Arthroscopy Association of North America: board or committee member; Elsevier: publishing royalties, financial or material support; Genzyme: paid presenter or speaker. Full ICMJE author disclosure forms are available for this article online, as supplementary material.

Received June 29, 2019; accepted July 16, 2020.

Address correspondence to Ian S. MacLean, M.D., 1611 W Harrison St., Suite 201, Chicago,IL 60612.E-mail: ian_s_maclean@rush.edu

(C) 2020 THE AUTHORS. Published by Elsevier Inc. on behalf of the Arthroscopy Association of North America. This is an open access article under the CC BY-NC-ND license (http://creativecommons.org/licenses/by-nc-nd/4.0/). 2666-061X/19792

https://doi.org/10.1016/j.asmr.2020.07.012 
acromial type classification has not been associated with RCTs or subacromial impingement, there is an association between the CSA and AI with RCTs. ${ }^{3}$ Both the CSA and AI are structural measurements and serve as proxies describing underlying anatomic variations among patients. Glenoid inclination, size of the humeral head relative to the acromion, and direction of the deltoid force vector relative to the glenohumeral articulation are all captured within these 2 metrics. ${ }^{4}$

A high CSA $\left(>35^{\circ}\right)$ has been associated with increased risk of RCT presumably due to the increased shear forces across the glenoid and greater stress placed on the rotator cuff. $^{2,4}$ Similarly, a high AI indicates a large lateral extension of the acromion and has been associated with RCTs. ${ }^{5-7}$ Conversely, a low CSA $\left(<30^{\circ}\right)$ has been associated with glenohumeral arthritis. ${ }^{1,8-10}$ One theory posits that this anatomy results in greater compressive forces across the glenohumeral articulation. ${ }^{1}$ Yet, this association with osteoarthritis has not been consistent across all studies. ${ }^{11}$

While a larger CSA and AI are associated with the development of RCTs, some studies suggest they do not correlate with poorer outcomes or risk of re-tear after rotator cuff repair (RCR). ${ }^{12,13}$ The literature is not uniform on the matter as other studies demonstrate greater risk of re-tear with variable effect on outcome measures.

Recent studies have examined the effect of acromioplasty on the CSA. A cadaveric study demonstrated that the CSA could be decreased by performing acromioplasty and further reduced with a lateral acromial resection. ${ }^{18}$ Significantly, this was noted to have no harmful effect on the deltoid origin. Clinical studies also have indicated that acromioplasty can decrease the CSA while also potentially reducing the risk of retear and increasing abduction strength. ${ }^{9-21}$ Notably, some suggest that an anterolateral acromioplasty, not just a lateral acromioplasty, also may decrease the CSA whereas other others debate the efficacy of a purely anterolateral acromioplasty. ${ }^{19,20}$

The purpose of this study was to evaluate the effect of acromioplasty using a cutting block technique on bony coverage as measured by the CSA and AI. The hypothesis was that acromioplasty would decrease these measures, whereas shoulders not undergoing acromioplasty would have no decrease in CSA or AI.

\section{Methods}

Institutional review board approval was obtained before initiation of this study. This was a retrospective review of patients recruited for a previous prospective randomized clinical trial looking at outcomes in patients with repair of a full-thickness tear of the superior cuff randomized to acromioplasty or no acromioplasty. ${ }^{22}$ The study offered enrollment to patients aged 18 years or older with a full-thickness tear of the superior rotator cuff between October 2007 and January 2011. The 2-year clinical outcomes of this study were previously published. During initial screening, exclusion criteria were applied to individuals with the following conditions: (1) isolated subscapularis tear, (2) irreparable or partially repairable RCTs after arthroscopic mobilization, (3) revision surgery.

After informed written consent, each patient was allocated to either the acromioplasty or non-acromioplasty treatment arms in a blinded fashion. Block randomization occurred through use of a sealed opaque envelope. Age, sex, marital status, occupation, workers compensation status, hand dominance, alcohol consumption, tobacco use, diabetes, and family history of RCT were extracted from the medical record. Furthermore, tear chronicity and acromial morphology according to Bigliani et al. ${ }^{23}$ were evaluated preoperatively by a fellowshiptrained sports medicine orthopaedic surgeon and recorded.

Between January 2017 and December 2017, patients were contacted for repeat follow-up clinical evaluation. Further exclusions were performed for interval patient death, advanced neurodegenerative conditions (e.g., Alzheimer dementia), and/or subsequent ipsilateral shoulder arthroplasty for advancing glenohumeral arthritis. Average time to first available postoperative radiograph was $17.0 \pm 28.9$ months after preoperative radiograph and $14.3 \pm 28.2$ months after surgery. Preand postoperative measurements of AI and CSA were performed by a blinded single reviewer as described by Nyffeler et al. and Moor et al (Fig 1). ${ }^{1,2}$ The blinded reviewer was a research assistant (A.K.G) trained to measure AI and CSA.

\section{Surgical Technique and Rehabilitation}

After tear confirmation, arthroscopic RCR was performed with the patient in the beach chair position by 1 of 4 subspecialty-trained, senior surgeons at a single center using their preferred single- or double-row technique. Tendon involvement, tear size, tear pattern, degree of retraction, tendon quality, and concomitant procedures were recorded, and type and number of anchors and repair configuration also were annotated.

For patients randomized to the acromioplasty group, the undersurface of the acromion was stripped of all bursal tissue and the coracoacromial ligament was released at its acromial attachment with a combination of shaver and/or radiofrequency wand. Once sufficiently exposed and viewed from the lateral portal, a motorized burr was then introduced from the posterior portal to remove bone to flat acromial undersurface using a cutting block technique. ${ }^{24}$

\section{Statistical Analysis}

Averages with standard deviation were calculated. Continuous variables were analyzed using independent 


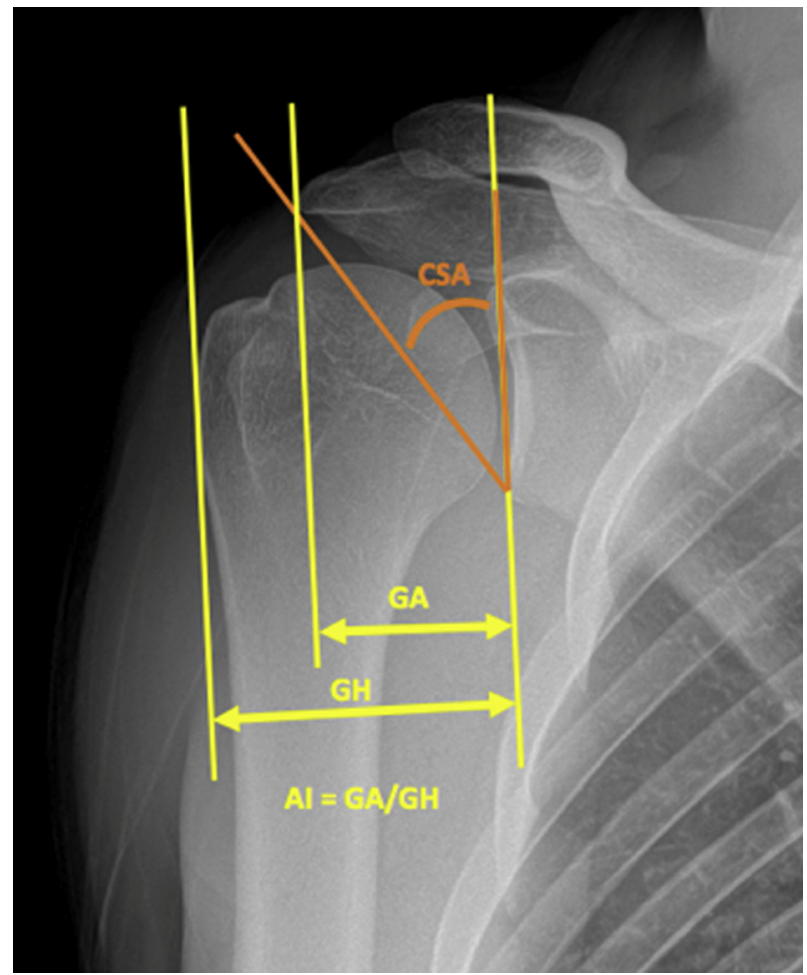

Fig 1. Measurement of CSA and AI from anteroposterior right shoulder radiograph. (AI, acromial index; CSA, critical shoulder angle; GA, distance between the glenoid plane and a parallel plane tangent to the lateral edge of the acromion; $\mathrm{GH}$, distance between the glenoid plane and a parallel plane tangent to the lateral edge of the humerus.). Reprinted with permission from the Journal of Shoulder and Elbow Surgery.

samples $t$ test, and dichotomous variables were compared using $\chi^{2}$ testing. A $P$ value of less than .05 with Bonferroni correction was deemed significant in the current study. Further, post-hoc power analysis was performed and determined that the present study is underpowered (power $=47.7 \%$ ). To demonstrate statistical significance with power of $80 \%$ and alpha of 0.05 , a sample size of 156 total patients would be required whereas only 71 were available for follow-up.

\section{Results}

After exclusion of 24 patients due to lack of follow up or exclusion, 71 patients $(75 \%)$ were available for analysis (Fig 2). Baseline demographics and acromial type demonstrated no statistically significant differences among patients in the acromioplasty and nonacromioplasty groups (Table 1).

When compared with preoperative measures, acromioplasty did not result in significant reductions in mean CSA $\left(34.5^{\circ}\right.$ vs $\left.35.5^{\circ} ; P=.293\right)$ or $\mathrm{AI}(0.68$ vs $0.66 ; P=.283)$ (Figs 3 and 4 ). Furthermore, postoperative CSA $\left(34.5^{\circ}\right.$ vs $\left.36.2^{\circ}, P=.052\right)$ and AI (0.66 vs $\left.0.67, P=.535\right)$ demonstrated no statistically significant differences between patients with and without acromioplasty, respectively.

\section{Discussion}

The hypothesis that acromioplasty using a traditional cutting block technique reduces acromial coverage was not supported by our data. In addition, there was no significant postoperative difference in the CSA and AI between the acromioplasty and no-acromioplasty groups.

The reported effect of acromioplasty on outcomes after RCR has been consistent in the literature with numerous prospective, controlled trials suggesting little to no effect of risk of retear or reported outcomes. ${ }^{22,25-27}$ The literature has been less consistent with regard to whether or not a high preoperative CSA and AI have any effect on RCR outcomes despite their association with initial tears. ${ }^{13-15}$ Even among those studies that do report an increased risk of retear, there are some that find this has no effect on patient reported outcomes. ${ }^{12,13,21}$ Although there may be an increased risk of radiographic retear with a greater postoperative CSA, clear reporting on retears of clinical significance and patient-reported outcomes is lacking. ${ }^{21}$ CSA and AI may simply be correlative of shoulder pathology and do not necessarily represent a modifiable risk factor. Or, as suggested by our study, CSA and AI may not be affected by acromioplasty at all.

In the midst of this uncertainty, potential "at-risk" acromial morphology has been viewed as a modifiable risk factor even without clear evidence to suggest it affects outcomes. In contrast to our study, a few cadaveric and clinical studies have demonstrated decreased CSA and AI following acromioplasty. ${ }^{18,19,21}$ This may be due

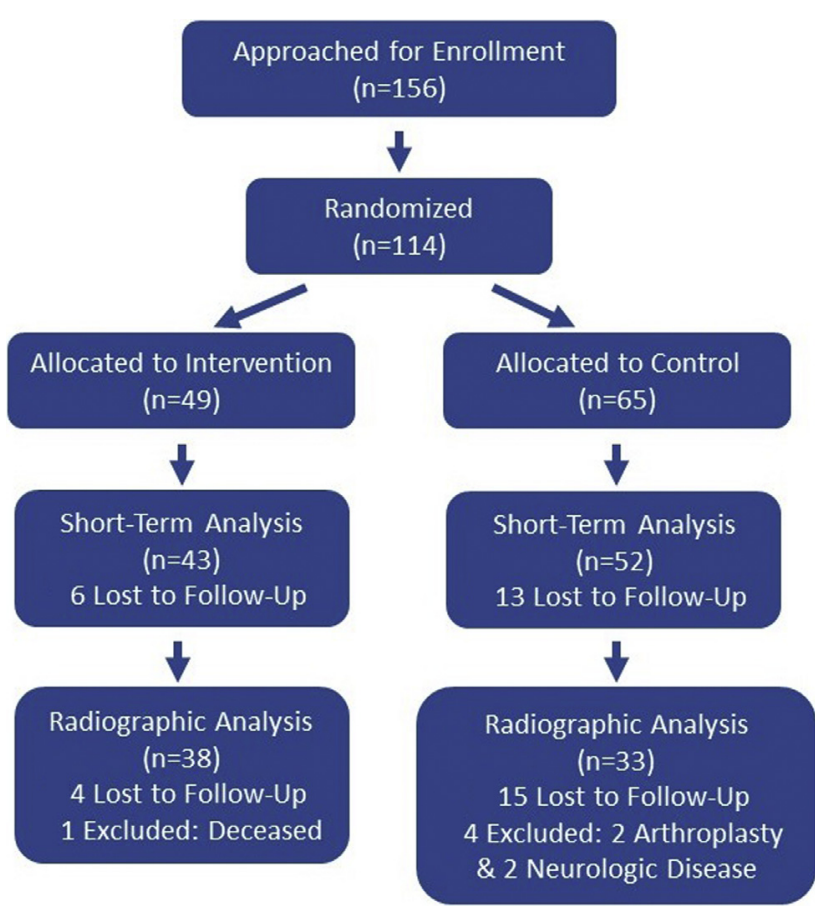

Fig 2. Patient allocation throughout the study. 
Table 1. Demographics of Patients Receiving Acromioplasty Versus No Acromioplasty

\begin{tabular}{lccc}
\hline & No & & \\
& Acromioplasty & Acromioplasty & $P$ Value \\
\hline $\mathrm{N}$ & 33 & 38 & \\
Mean age, y & 58.4 (SD 9.3) & 57.6 (SD 9.1) & .732 \\
Sex, M:F & $21: 11$ & $25: 13$ & .849 \\
Smoker, \% & $12 \%$ & $15 \%$ & 1.000 \\
Diabetes, \% & $0 \%$ & $5 \%$ & .894 \\
WC & $19 \%$ & $20 \%$ & .660 \\
Acromial morphology & I: 3 & I: 2 & .142 \\
& II: 12 & II: 14 & \\
Mean preoperative AI & III: 3 & III: 9 & \\
Mean postoperative AI & 0.67 (SD 0.09) & 0.68 (SD 0.09) & .633 \\
Mean preoperative CSA & 37.5 (SD 4.7) & 35.5 (SD 4.4) & .143 \\
Mean postoperative CSA & 36.2 (SD 3.7) & 34.5 (SD 3.8) & .073 \\
\hline
\end{tabular}

NOTE. I indicates Bigliani type 1 acromion: flat; II indicates Bigliani type 2 acromion: curved; and III indicates Bigliani type 3 acromion: hooked.

AI, acromial index; CSA, critical shoulder angle; F, female; M, male; SD, standard deviation; WC, workers compensation.

to differences in technique or more aggressive debridement and resection of the acromion.

One consideration is that the decompression provided by the cutting block technique does not address the area of bone contributing to the measures of CSA and AI as it may not get far enough lateral. However, a study by Billaud et al. ${ }^{19}$ reported a statistically significant decrease in the CSA with arthroscopic anterolateral acromial resection. Postoperatively, they had a $34 \%$ decrease in the number of patients with a CSA $>35^{\circ}$. A recent cadaveric computed tomography imaging study identified the "critical acromial point," the point primarily responsible for the acromial contribution to the CSA, at $21 \%$ of the anterior to posterior length from the anterolateral corner. ${ }^{28}$ This places the critical acromial point within the area in which it would be decompressed by an anterolateral decompression using a cutting block technique. Yet, with direct comparison of techniques, it seems that a lateral acromioplasty may be more reliable and the anterolateral acromioplasty more vulnerable to scapular morphology variances. ${ }^{20}$

While there is debate on whether an increased CSA affects risk of retear and outcome measures, other studies question the reliability of the CSA and if it should even be used. Radiographic technique is highly important, and small changes in projection can significantly alter the apparent CSA. ${ }^{29}$ In evaluating more than 1500 radiographs, Chalmers et al. $^{30}$ found that only $57 \%$ met quality criteria. In addition, while they found a high mean CSA in patient with degenerative cuff tears, the difference was small and could have been due to measurement error.

There are several strengths of this study. This study included patients from multiple surgeons specialized in shoulder surgery. Therefore, results are less likely to be biased due to one surgeon's technique and are generalizable to other busy sports and shoulder practices. Our patient population is drawn from a standard population seen in a major metropolitan area and is therefore likely representative of other practices. In addition, the cutting block technique is commonly used for acromioplasty and increases the generalizability of the study.

\section{Limitations}

This study does have a several limitations. Our sample size is small, with 71 patients available for follow-up.

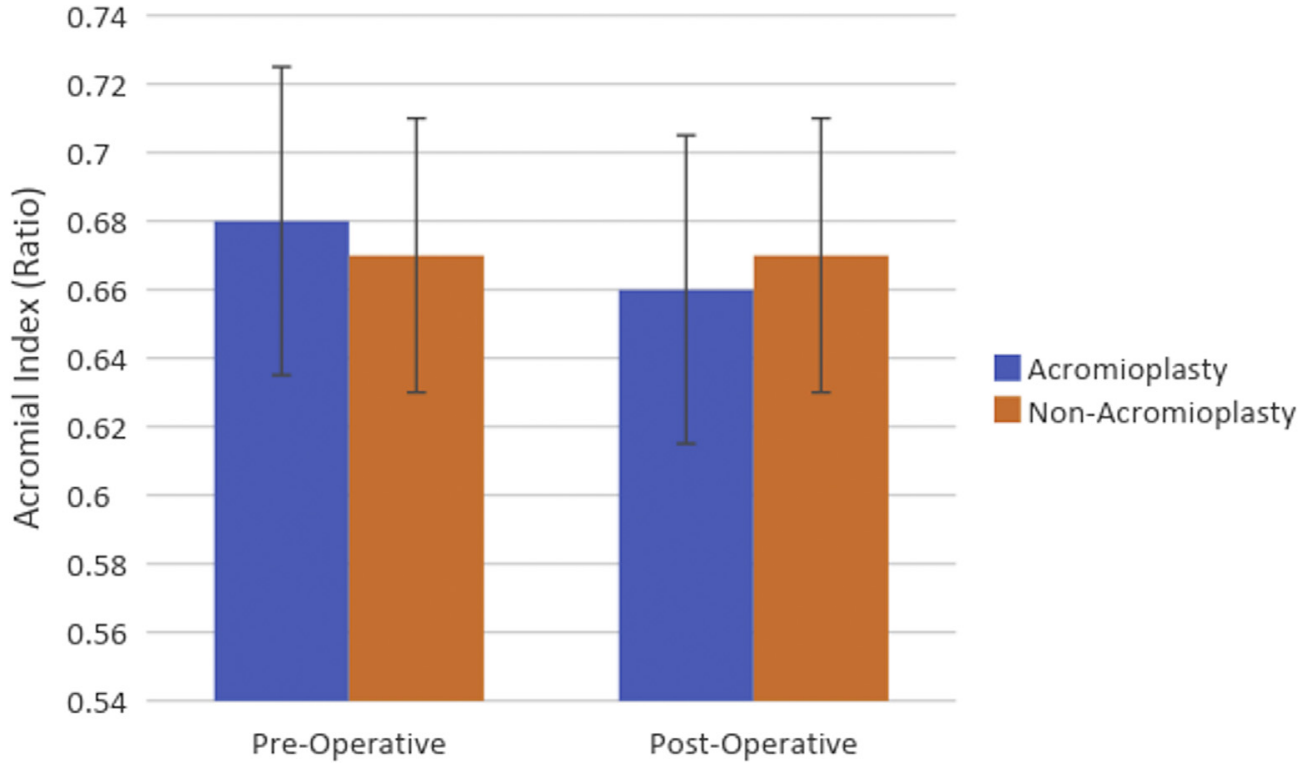

Fig 3. Effect of acromioplasty on acromial index. 


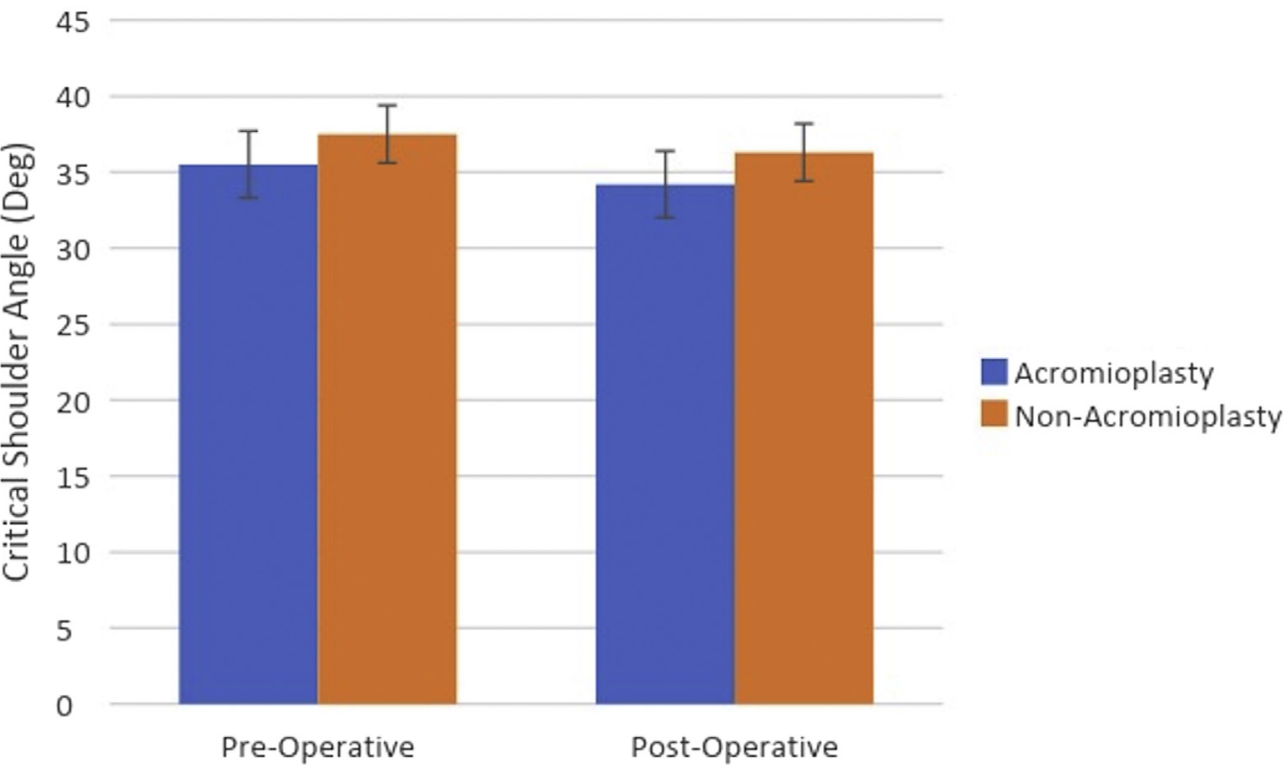

This represents $62 \%$ of the initial 114 patients who were randomized with a greater number failing to follow up from the no acromioplasty group. As a result, the study is underpowered, and this may increase the chance of type II error. In addition, we attempt to describe small changes in the CSA. Yet, it has previously been demonstrated that small variations in patient positioning during radiographic exam can affect this measurement. ${ }^{29,30}$ However, this study did not collect data assessing for radiograph quality. Finally, although patients who underwent shoulder arthroplasty were excluded from the final analysis, degree of degenerative changes, if any, were not described. This creates a selection bias because degenerative change could result in changes in AI and CSA.

\section{Conclusions}

There was no statistically significant change in either the CSA or AI following acromioplasty, nor was there a significant postoperative difference in CSA or AI between the group that underwent acromioplasty and the group that did not.

\section{References}

1. Moor BK, Bouaicha S, Rothenfluh DA, Sukthankar A, Gerber C. Is there an association between the individual anatomy of the scapula and the development of rotator cuff tears or osteoarthritis of the glenohumeral joint? A radiological study of the critical shoulder angle. Bone Joint J 2013;95-b:935-941.

2. Nyffeler RW, Werner CM, Sukthankar A, Schmid MR, Gerber C. Association of a large lateral extension of the acromion with rotator cuff tears. J Bone Joint Surg Am 2006;88:800-805.
3. Balke M, Schmidt C, Dedy N, Banerjee M, Bouillon B, Liem D. Correlation of acromial morphology with impingement syndrome and rotator cuff tears. Acta Orthop 2013;84:178-183.

4. Li X, Olszewski N, Abdul-Rassoul H, Curry EJ, Galvin JW, Eichinger JK. Relationship between the critical shoulder angle and shoulder disease. JBJS Rev 2018;6:el.

5. Moor BK, Wieser K, Slankamenac K, Gerber C, Bouaicha S. Relationship of individual scapular anatomy and degenerative rotator cuff tears. J Shoulder Elbow Surg 2014;23:536-541.

6. Ames JB, Horan MP, Van der Meijden OA, Leake MJ, Millett PJ. Association between acromial index and outcomes following arthroscopic repair of full-thickness rotator cuff tears. J Bone Joint Surg Am 2012;94:1862-1869.

7. Torrens C, Lopez JM, Puente I, Caceres E. The influence of the acromial coverage index in rotator cuff tears. J Shoulder Elbow Surg 2007;16:347-351.

8. Heuberer PR, Plachel F, Willinger L, et al. Critical shoulder angle combined with age predict five shoulder pathologies: A retrospective analysis of 1000 cases. BMC Musculoskelet Disord 2017;18:259.

9. Mantell MT, Nelson R, Lowe JT, Endrizzi DP, Jawa A. Critical shoulder angle is associated with full-thickness rotator cuff tears in patients with glenohumeral osteoarthritis. J Shoulder Elbow Surg 2017;26:e376-e381.

10. Spiegl UJ, Horan MP, Smith SW, Ho CP, Millett PJ. The critical shoulder angle is associated with rotator cuff tears and shoulder osteoarthritis and is better assessed with radiographs over MRI. Knee Surg Sports Traumatol Arthrosc 2016;24:2244-2251.

11. Shinagawa K, Hatta T, Yamamoto N, et al. Critical shoulder angle in an East Asian population: Correlation to the incidence of rotator cuff tear and glenohumeral osteoarthritis. J Shoulder Elbow Surg 2018;27:1602-1606.

12. Kirsch JM, Nathani A, Robbins CB, Gagnier JJ, Bedi A, Miller BS. Is there an association between the "critical 
shoulder angle" and clinical outcome after rotator cuff repair? Orthop J Sports Med 2017;5:2325967117702126.

13. Lee M, Chen JY, Liow MHL, Chong HC, Chang P, Lie D. Critical shoulder angle and acromial index do not influence 24-month functional outcome after arthroscopic rotator cuff repair. Am J Sports Med 2017;45:2989-2994.

14. Garcia GH, Liu JN, Degen RM, et al. Higher critical shoulder angle increases the risk of retear after rotator cuff repair. J Shoulder Elbow Surg 2017;26:241-245.

15. Li H, Chen Y, Chen J, Hua Y, Chen S. Large critical shoulder angle has higher risk of tendon retear after arthroscopic rotator cuff repair. Am J Sports Med 2018;46:1892-1900.

16. Scheiderer B, Imhoff FB, Johnson JD, et al. Higher critical shoulder angle and acromion index are associated with increased retear risk after isolated supraspinatus tendon repair at short-term follow up. Arthroscopy 2018;34: 2748-2754.

17. Sheean AJ, Sa D, Woolnough T, Cognetti DJ, Kay J, Burkhart SS. Does an increased critical shoulder angle affect re-tear rates and clinical outcomes following primary rotator cuff repair? A systematic review. Arthroscopy 2019;35:2938-2947.e2931.

18. Katthagen JC, Marchetti DC, Tahal DS, Turnbull TL, Millett PJ. The effects of arthroscopic lateral acromioplasty on the critical shoulder angle and the anterolateral deltoid origin: an anatomic cadaveric study. Arthroscopy 2016;32:569-575.

19. Billaud A, Cruz-Ferreira E, Pesquer L, Abadie P, Carlier Y, Flurin PH. Does the critical shoulder angle decrease after anterior acromioplasty? Arch Orthop Trauma Surg 2019;139:1125-1132.

20. Kaiser D, Bachmann E, Gerber C, Meyer DC. Influence of the site of acromioplasty on reduction of the critical shoulder angle (CSA) - an anatomical study. BMC Musculoskelet Disord 2018;19:371.

21. Gerber C, Catanzaro S, Betz M, Ernstbrunner L. Arthroscopic correction of the critical shoulder angle through lateral acromioplasty: A safe adjunct to rotator cuff repair. Arthroscopy 2018;34:771-780.

22. Abrams GD, Gupta AK, Hussey KE, et al. Arthroscopic repair of full-thickness rotator cuff tears with and without acromioplasty: Randomized prospective trial with 2-year follow-up. Am J Sports Med 2014;42:1296-1303.

23. Bigliani LU, Morrison DS, April EW. The morphology of the acromion and its relationship to rotator cuff tears. Orthop Trans 1986;10.

24. Caspari RB, Thal R. A technique for arthroscopic subacromial decompression. Arthroscopy 1992;8:23-30.

25. Milano G, Grasso A, Salvatore M, Zarelli D, Deriu L, Fabbriciani C. Arthroscopic rotator cuff repair with and without subacromial decompression: A prospective randomized study. Arthroscopy 2007;23:81-88.

26. MacDonald P, McRae S, Leiter J, Mascarenhas R, Lapner P. Arthroscopic rotator cuff repair with and without acromioplasty in the treatment of full-thickness rotator cuff tears: a multicenter, randomized controlled trial. J Bone Joint Surg Am 201 1;93:1953-1960.

27. Gartsman GM, O'Connor DP. Arthroscopic rotator cuff repair with and without arthroscopic subacromial decompression: A prospective, randomized study of oneyear outcomes. J Shoulder Elbow Surg 2004; 13:424-426.

28. Karns MR, Jacxsens M, Uffmann WJ, Todd DC, Henninger HB, Burks RT. The critical acromial point: the anatomic location of the lateral acromion in the critical shoulder angle. J Shoulder Elbow Surg 2018;27:151-159.

29. Suter T, Gerber Popp A, Zhang Y, Zhang C, Tashjian RZ, Henninger HB. The influence of radiographic viewing perspective and demographics on the critical shoulder angle. J Shoulder Elbow Surg 2015;24:e149-e158.

30. Chalmers PN, Salazar D, Steger-May K, Chamberlain AM, Yamaguchi K, Keener JD. Does the critical shoulder angle correlate with rotator cuff tear progression? Clin Orthop Relat Res 2017;475:1608-1617. 